\title{
Suaeda maritima (L.) Dumort GREEN SYNTHESIS- REDUCED NICKEL OXIDE NANOPARTICLES FOR ANTIOXIDANT AND BACTERICIDAL ACTIVITY
}

\author{
T. Krishnasree ${ }^{1 *}$, Pavani Peddi ${ }^{2}$ \\ ${ }^{1}$ Department of Freshman Engineering, PVP Siddhartha Institute of Technology, Kanuru, Vijayawada, AP, India \\ ${ }^{2}$ Department of Chemistry, FED, PVP Siddhartha Institute of Technology, Kanuru-520007, Vijayawada, AP, India
}

Received - August 18, 2021; Revision - November 06, 2021; Accepted - December 16, 2021

Available Online - December 30, 2021

DOI: http://dx.doi.org/10.18006/2021.9(6).823.830

\section{KEYWORDS \\ Suaeda maritima (L.) Dumort}

Green synthesis

$\mathrm{NiO}$ nanoparticles

SEM

XRD

Biological activity

\begin{abstract}
A unique way, green, cost-effective, and direct fabrication method is proposed for the synthesis of Nickel Oxide Nanoparticles (NPs) in an eco-environmentally way through leaf extract of Suaeda maritima (L.) Dumort. The nickel oxide nanoparticles were synthesized using Nickel (II) nitrate hexahydrate as a metal source and aqueous leaf extract of S. maritima was utilized as a green reducing agent. The formation of NPs was monitored by the change in color in the reaction mixture and the synthesized NPs were characterized using UV-visible spectrophotometer, Fourier Transform infrared (FT-IR) spectroscopy, field emission scanning electron microscope (FE SEM), X-ray diffractometer (XRD), and energy-dispersive Xray spectroscopy (EDX). Further, the antibacterial activity of synthesized NPs was carried using the agar plate well diffusion method and antioxidant activity by DPPH free radical scavenging activity of the NPs was studied. The UV-visible absorption spectra of nanoparticles show a characteristic maximum absorption peak centered at $397 \mathrm{~nm}$. The functional group analysis by FT-IR confirms the presence of various bioactive functional groups in the synthesized particles. The structural characterization confirms that the particles were Face Centred Cubic lattice structure having IR-regular in shape and rough surface with average atomic weight percentages of $76.3 \%$. The synthesized nanoparticles were found to be potent against the growth of gram-positive (Bacillus subtilis, Staphylococcus aureus) and gram-negative (Escherichia coli, Pseudomonas aeruginosa) bacteria. In the DPPH assay, the IC 50 values of the synthesized NPs were found to be $28.01 \mu \mathrm{g} / \mathrm{mL}$ which is very close to standard ascorbic acid (22.19 $\mu \mathrm{g} / \mathrm{mL}$ ) whereas the IC 50 of the aqueous plant leaf extract was found to be $47.30 \mu \mathrm{g} / \mathrm{m}$ confirms that the nanoparticles having enhanced antioxidant activity. From the results of the study it can be concluded that this protocol is simple, rapid, one step, eco-friendly, non-toxic for the synthesis of nickel nanoparticles.
\end{abstract}

* Corresponding author

E-mail: krishnasreephysics@gmail.com (T. Krishnasree)

Peer review under responsibility of Journal of Experimental Biology and Agricultural Sciences.

Production and Hosting by Horizon Publisher India [HPI] (http://www.horizonpublisherindia.in/).

All rights reserved.
All the articles published by Journal of Experimental Biology and Agricultural Sciences are licensed under a Creative Commons Attribution-NonCommercial 4.0 International License Based on a work at www.jebas.org.

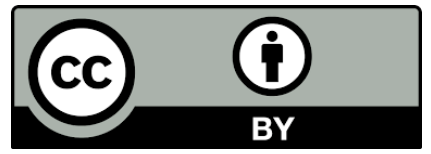




\section{Introduction}

Nanotechnology is a branch of science that dealt with the matter having at least $1-100 \mathrm{~nm}$ one dimension size and having the ability to engineer material by controlling its particle size (Palasantzas et al., 2008; Stark et al., 2015). The significant economic importance in the field of magnetic, electronic, and catalytic application of Nickel $(\mathrm{Ni})$ and nickel oxide $(\mathrm{NiO})$ nanoparticles have gained specific interest than the NPs of other noble metals (Cristino et al., 2020; Taiba \& Tayyiba, 2021). The Ni NPs and NiO NPs have super capacitance properties, electrocatalysis, and electron transfer capability, along with this, these NPs are having high chemical stability. Further, NPs have several applications in biomedicines such as anti-inflammatory, antioxidant, and antibacterial agents (Anandgaonker et al., 2019; Haider et al., 2020). Top-down and bottom-up methods are the two fundamental principles utilized for the synthesis of NPs of various sizes, shapes, and applications. The NPs are synthesized by utilizing simpler molecules in a bottom-up approach. In this chemical vapor deposition, spray pyrolysis, laser pyrolysis, condensation, and sol-gel processes were utilized for the synthesis of NPs (Huston et al., 2021). But the synthesis of NPs in these techniques has various limitations like stability in a hostile environment; requiring specialized technical knowledge, expansive, bioaccumulation, and toxicity.

To eliminate the limitations in the synthesis of NPs using chemical methods, green synthesis approaches are gaining more attention in the development of NPs of various applications. In green synthesis, NPs are synthesized through an environmentally friendly process by minimizing waste, utilization of non-toxic chemicals, reduction of derivatives as well as renewable feedstock (Singh et al., 2018). In the synthesis of NPs by green methodology, the biological precursors at various strengths, volumes, $\mathrm{pH}$ ranges, and temperature were utilized. In these methods, the biodiversity of plants with various bioactive compounds were considered for the synthesis of NPs. The plant bioactive compounds are having the capability to reduce the metal salts into metal NPs and hence are utilized as reducing agents for the synthesis of NPs (Ibrahim et al., 2019).

The mangrove herb S. maritima (sea blite) belongs to the family Amaranthaceae and is locally called Elakura in Andhra Pradesh. It is distributed worldwide and grown in coastal salt flats and tidal wetlands near the sea. It is edible as a leaf vegetable used for making juice and curries, feeding cattle, goats, and sheep (Bandaranayake, 2002). In local traditional medicine, it is used for the treatment of Hepatitis and is having hepatoprotective (Ravi Kumar et al., 2011) antioxidant (Patra et al., 2011) and antimicrobial (Nayak et al., 2018) activities. Hence the present work is intended to synthesize NiO NPs through a single step and green method and evaluation of its antioxidant and antimicrobial activities.

\section{Materials and Methods}

\subsection{Chemicals and Materials}

Nickel (II) nitrate hexahydrate (99.8\%), 2,2-diphenyl-1picrylhydrazyl (DPPH), peptone, beef extract, agar, and other chemicals used in the study were of analytical grade. The leaves of the plant S. maritima, was collected in Mangrove Forest, near Gilakaladindi, Machilipatnam, Krishna district, AP. The leaves of the plant were shaded dried, powder, and preserved in an amber color bottle and are used during the synthesis of NPs using Ni as a metal precursor.

\subsection{Preparation of leaf extract}

An accurately weighed 2 grams of plant leaf powder was taken in a $500 \mathrm{~mL}$ volumetric flask containing $200 \mathrm{~mL}$ of distilled water. The content was boiled on a magnetic stirrer with a hot plate at $60{ }^{0} \mathrm{C}$ for 20 minutes. Then it was cooled, filtered through Whatman \#1 paper and the final volume made up to the mark in a $250 \mathrm{~mL}$ volumetric flask and the filtrate was used for the synthesis of $\mathrm{NiO}$ NPs.

\subsection{Preparation of metal solution}

The Nickel nitrate solution at a concentration of $1 \mathrm{M}$ was used for the synthesis of NPs. An accurately weighed 29.08 grams of Nickel (II) nitrate hexahydrate was dissolved in $100 \mathrm{~mL}$ distilled water and the solution was used for the synthesis of NPs.

\subsection{Synthesis of NiO NPs}

The synthesis of NiO NPs was carried as per the procedure described by Mohammed \& Olajire (2020) briefly, to $10 \mathrm{~mL}$ of Nickel nitrate solution and $10 \mathrm{~mL}$ of aqueous leaf extract of $S$. maritima, was added and mixed. Then the $\mathrm{pH}$ of the solution was adjusted to 12 using sodium hydroxide solution. The color change of the solution after the immediate addition of leaf extract to the metal solution was noted and the solution was stirred in a magnetic stirrer for 10 minutes. The change in color and the formation of $\mathrm{NiO}$ NPs were observed. The solution with NiO NPs was centrifuged and the obtained NPs were stored in a sample vial at room temperature.

\subsection{Characterization of NiONPs}

The synthesized NiO NPs were characterized using different techniques such as UV-visible spectrophotometer, Fourier transform infrared (FT-IR) spectroscopy, field emission scanning electron microscope (FE SEM), X-ray diffractometer (XRD), and energy-dispersive X-ray spectroscopy (EDX). The change in the color observed while converting the metal into metal oxide was confirmed by determining the optical absorption properties of the 
solution and was performed using a UV-visible spectrophotometer (JASCO, Japan) at 800 to $200 \mathrm{~nm}$ wavelength range. The type of functional groups that are involved in the reduction of metal and formation of NPs was determined by performing functional group analysis and was carried on FT-IR spectroscopy (Bruker, USA) in the range of 4000 to $500 \mathrm{~cm}^{-1}$. The morphological characteristics and size of the synthesized NPs were determined using FE SEM (Nova, Nanosem-450, FEI, USA). The crystal nature and the lattice structure of the synthesized NPs were determined by XRD (Rigaku Corporation, Japan) and is carried at a scan speed of $2^{0} / \mathrm{min}$ in the diffraction angles $(2 \theta)$ from $20^{\circ}$ to $80^{\circ}$. The EDX (RONTEC's, Model QuanTax 200, Germany) analysis in the range of 0-17 KeV was performed to evaluate the elemental composition of the synthesized NPs (Gokce et al., 2012; Pasupuleti et al., 2013)

\subsection{Antibacterial activity of synthesized NiO NPs}

The application of the synthesized NiO NPs as a bactericidal agent was confirmed by performing anti-bacterial activity against two gram-positive bacteria namely B. subtilis (MTCC - 1427) and $S$. aureus (MTCC - 1430), two gram-negative bacteria namely E. coli (MTCC - 294) and P. aeruginosa (MTCC - 1748). Agar plate well diffusion method based on the procedure described by Priyanka et al. (2019) was performed to evaluate the antibacterial activity of NiO NPs. Briefly, the bacterial seeded medium was prepared by mixing $100 \mathrm{~mL}$ of medium and $1 \mathrm{~mL}$ of $10^{7} \mathrm{CFU}$ selected bacteria to attain $10^{5} \mathrm{CFU} / \mathrm{ml}$ of medium. Then in a sterile petri dish, 10 $\mathrm{mL}$ of nutrient agar medium was poured as a basal layer followed by $15 \mathrm{~mL}$ of seeded medium. The plates were kept undisturbed till solidification of the medium in the Petri plate and wells were prepared using a sterilized stainless-steel cork borer. In each well, $25 \mu \mathrm{L}$ of selected concentrations of aqueous plant extract, standard gentamycin, and synthesized NPs were loaded separately with sterile micro-pipette. Water was served as a negative control in the study and the plates were incubated for bacteria growth at $37{ }^{\circ} \mathrm{C}$ for $24 \mathrm{hrs}$. Then the zone of inhibition of standard, NiO NPs, and aqueous plant extract was measured in $\mathrm{mm}$ by comparing with negative control.

\subsection{DPPH radical scavenging assay}

The antioxidant activity of synthesized NiO NPs was determined by performing DPPH free radical scavenging assay as per the procedure described by Akintola et al. (2020). To select concentrations of aqueous plant extract and the synthesized NPs solutions, an equal volume of methanolic DPPH $(0.135 \mathrm{~mm})$ solution was added and incubated at room temperature for 30 minutes. Then the radical scavenging activity of the NPs and plant extract was monitored at $517 \mathrm{~nm}$ using a UV-visible spectrophotometer. The absorbance obtained at $517 \mathrm{~nm}$ for plant extract and NPs was used for calculating the percentage DPPH inhibition and 50 percent inhibitory concentrations ( $\left.\mathrm{IC}_{50}\right)$ separately.

\section{Results and Discussion}

Simple cost-effective green syntheses of NiO NPs were effectively synthesized using $S$. maritima, leaf extract as a biological reducing agent. The process and action mechanism of synthesized NiO NPs were summarized in figure 1.

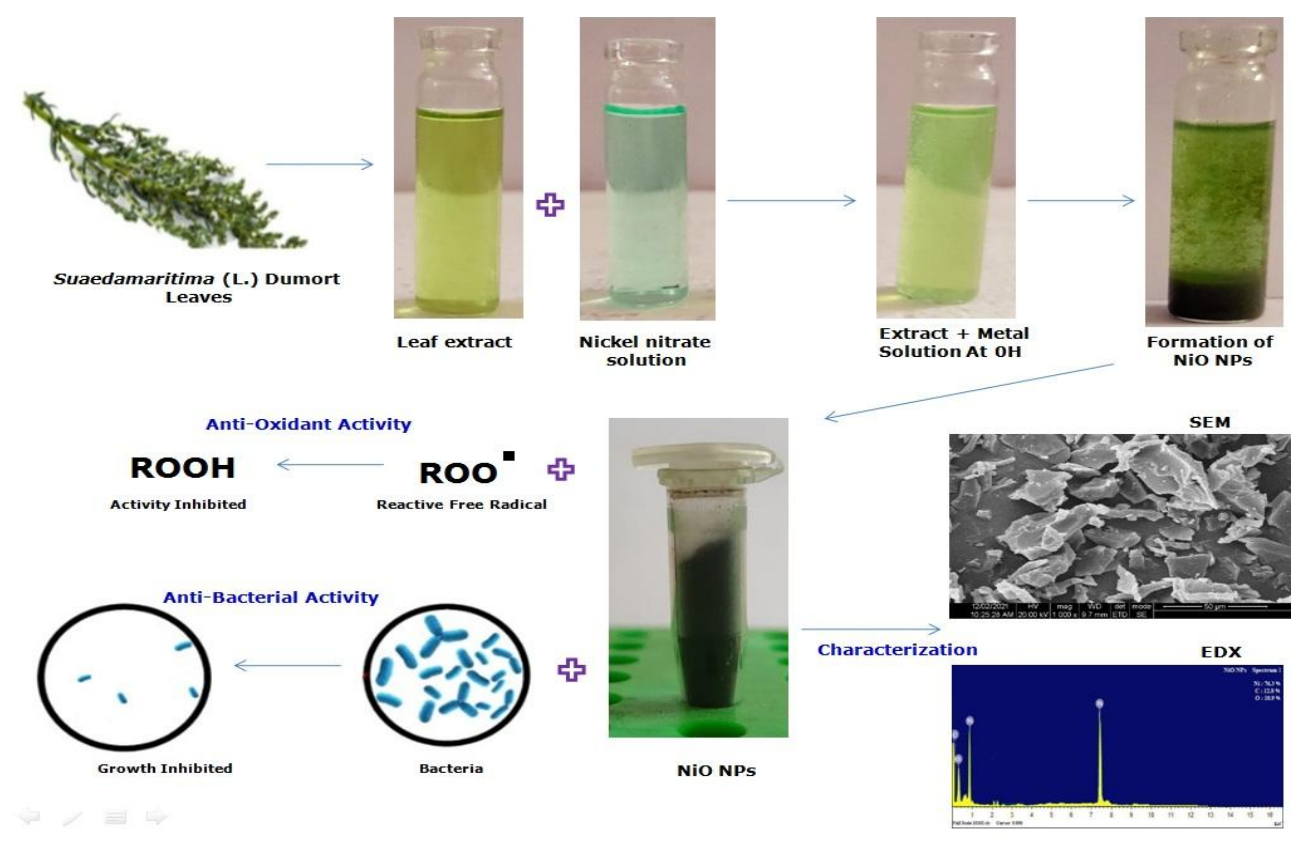

Figure 1 The process of synthesis of NiO NPs

Journal of Experimental Biology and Agricultural Sciences

http://www.jebas.org 

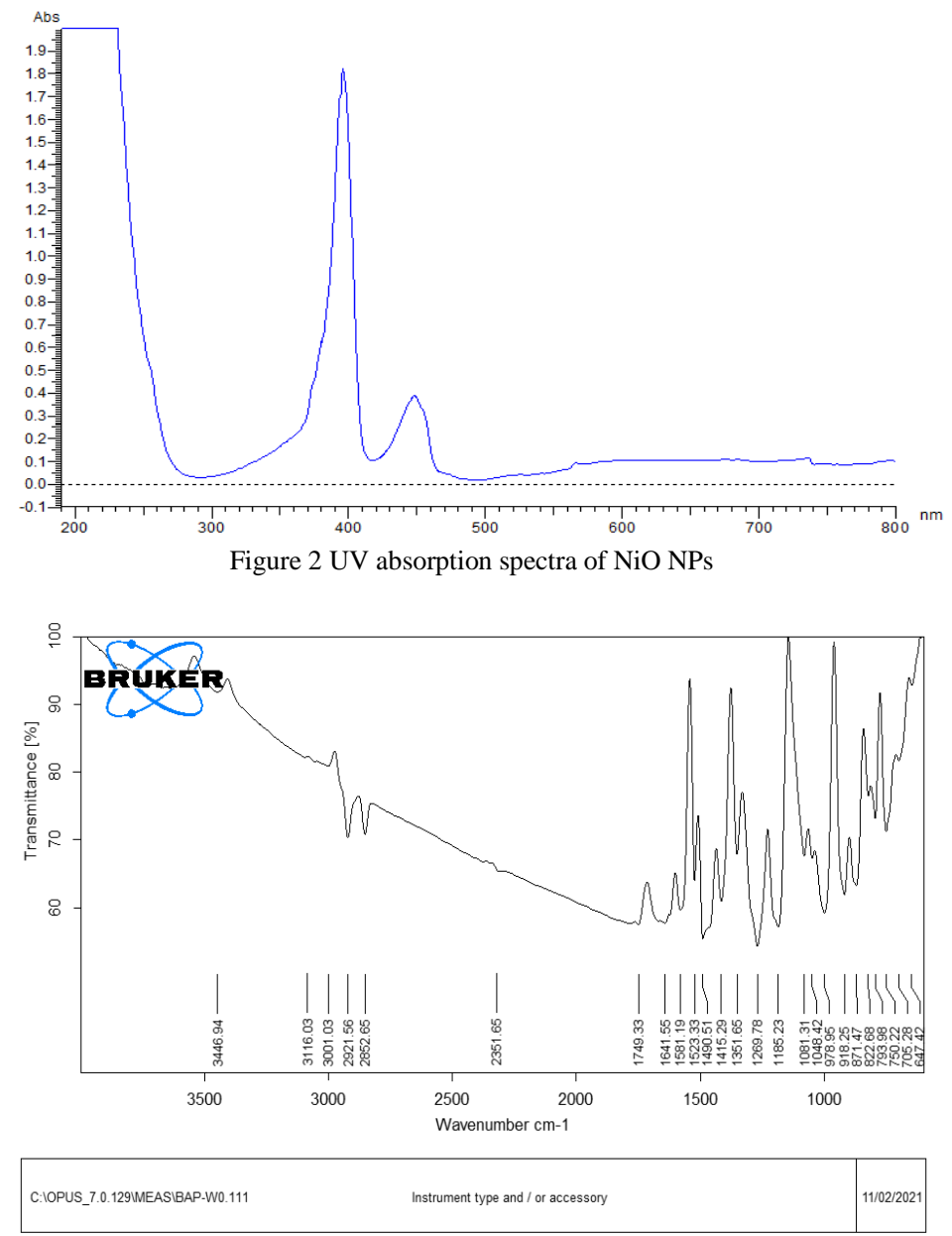

Figure 3 FT-IR spectrum of NiO NPs

\subsection{Optical absorption study}

The UV-visible absorption spectra of NiO NPs show a characteristic maximum absorption peak centered at $397 \mathrm{~nm}$ (Figure 2). In the absorption spectra, the wavelength curve observed at the $440 \mathrm{~nm}$ range corresponds to the surface plasmon resonance of nickel (Khalid et al., 2011). The absorption maxima and bandwidth of the surface plasmon resonance were depend on the shape and size of the NPs. The broadness in the peak indicates the small size of the NPs (Evanoff \& Chumanov, 2005) and hence is evidence of the formation of less particle size of the NPs. The UV characteristic absorption in this finding was in good correlation with the reported studies confirms that the characteristic wavelength absorbed in this study was due to the formation of NiO NPs (Pandian et al., 2015; Singh et al., 2018)

\subsection{Functional group analysis by FT-IR spectroscopy}

The possible bioactive compounds of the $S$. maritima aqueous leaves extract that are involved in the reduction of $\mathrm{Ni}$ metal and formation of NiO NPs were determined by FT-IR spectroscopy analysis. The FT-IR transmittance bands are found at $3446 \mathrm{~cm}^{-1}$ and $3116 \mathrm{~cm}^{-1}$ corresponding to $\mathrm{O}-\mathrm{H}$ stretching in free and intramolecular bonded alcohol respectively. The transmittance peak at $3001 \mathrm{~cm}^{-1}$ corresponds to $\mathrm{O}-\mathrm{H}$ stretching in a carboxylic acid. The FT-IR transmittance peak at $2921 \mathrm{~cm}^{-1}$ and $2852 \mathrm{~cm}^{-1}$ is due to the presence of N-H stretch in the amine group and C-H stretch in the aldehyde group. A strong and intense band at 1641 $\mathrm{cm}^{-1}$ corresponds to $\mathrm{C}=\mathrm{C}$ monosubstituted alkenes. Strong bond at $1749 \mathrm{~cm}^{-1}, 1581 \mathrm{~cm}^{-1}$, and $1269 \mathrm{~cm}^{-1}$ correspond to $\mathrm{C}-\mathrm{H}$ bending, $\mathrm{C}=\mathrm{C}$ stretching, and $\mathrm{C}-\mathrm{N}$ stretch vibrations in aromatic compounds (Mude et al., 2009; Wang et al., 2014). The intense band at $705 \mathrm{~cm}^{-1}$ can be associated with $\mathrm{Ni}-\mathrm{O}-\mathrm{H}$ bending and $\mathrm{Ni}-\mathrm{O}$ stretching vibration in NiO NPs (Ebin, 2018). The metal-oxygen (Ni-O) stretching frequency of the synthesized NPs was identified by the presence of an intense peak at $978 \mathrm{~cm}^{-1}$ (Suresh et al., 2016). The results of FT-IR analysis prove that the presence of a different type of bioactive phytoconstituents in the leaf extract of S. maritima is responsible for the reduction of $\mathrm{Ni}$ and the formation of NiO NPs. (Figure 3) 


\subsection{Structural characterization}

HR-SEM analysis of the synthesized NiO NPs confirms the surface morphology and size of the NPs and the SEM image was given in figure 4 . The NPs are uniformly distributed in size with an aggregated arrangement of particles. The NPs were observed to be irregular in shape with a rough surface and the particles are 10 to $49 \mathrm{~nm}$ size range. Similar types of NPs with a similar degree of agglomerations were identified by Zahra et al. (2021).

The EDX analysis of the synthesized Ni NPs was performed to ensure the Ni content in the NPs and the EDX spectrum was given in figure 5. The typical EDX spectrum confirms the presence of pure $\mathrm{Ni}$ in a single phase. The presence of oxygen indicates that the $\mathrm{Ni}$ is in oxide form and the presence of carbon in the EDX spectra may be due to the plant bioactive compounds that are responsible for the reduction of $\mathrm{Ni}$. The $\mathrm{Ni}$ was identified at $\mathrm{K} \alpha$ of 7.4 and $L \alpha$ of 0.8 with an average atomic weight percentage of $76.3 \%$. The atomic weight percentages of carbon $(\mathrm{C}-\mathrm{K} \alpha)$ and oxygen $(\mathrm{O}-\mathrm{K} \alpha)$ was found to be 12.8 and 10.9 percent respectively. The percentage metal content in the present study was in correlation with the findings reported by Zahra et al. (2021) but the size of the NPs was very less in the present study than the reported study.

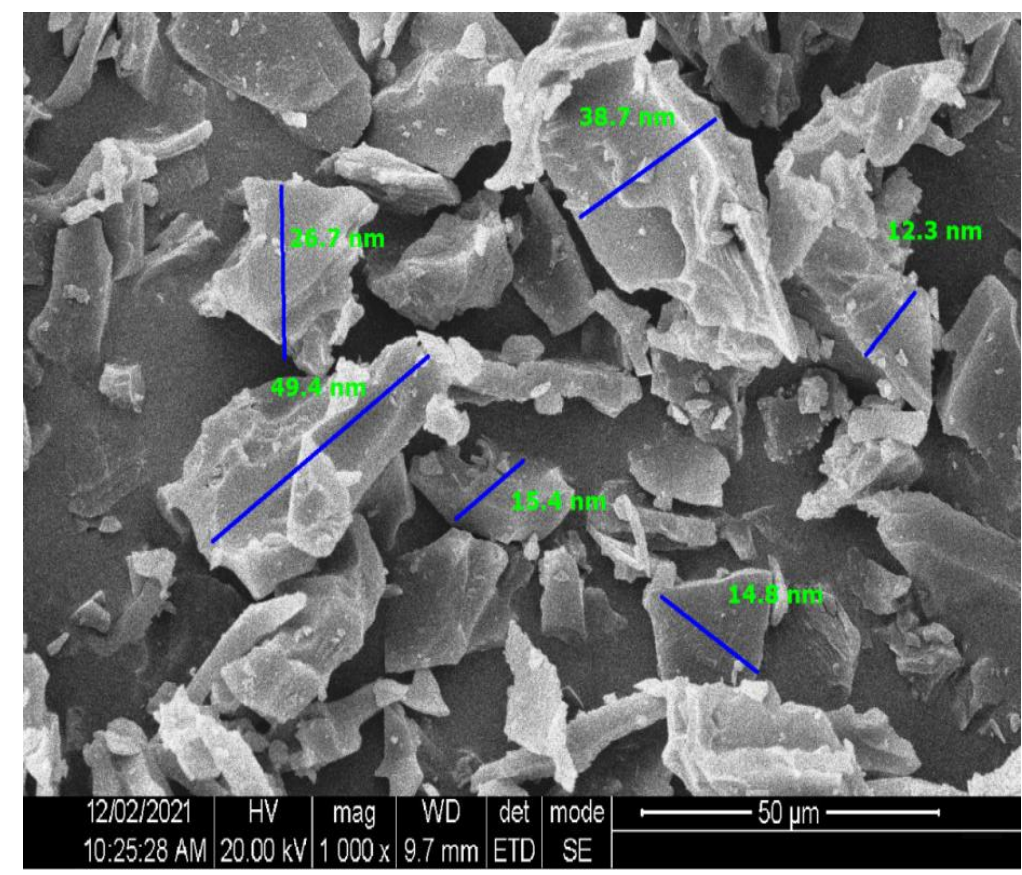

Figure 4 SEM image of synthesized NiO NPs

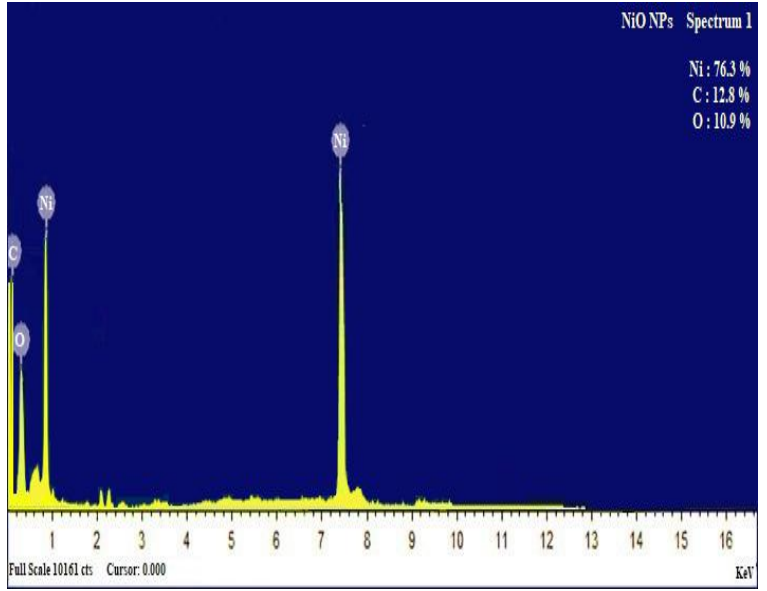

Figure 5 EDX spectra of the synthesized NiO NPs

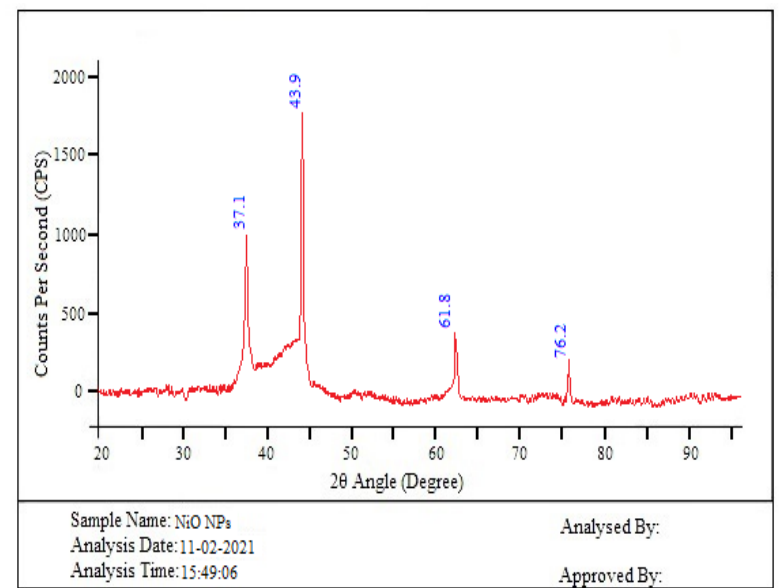

Figure 6 XRD spectra of the synthesized NiO NPs

Journal of Experimental Biology and Agricultural Sciences http://www.jebas.org 
The crystallinity, particle size, and phase composition of the synthesized NiO NPs were confirmed by XRD analysis and the XRD spectra was given in figure 6 . The XRD diffraction spectra show peaks at $2 \theta$ values of $37.1^{\circ}, 43.9^{\circ}, 61.9^{\circ}$ and $76.2^{\circ}$ corresponds to (111), (200), (220), and (311) planes which are in correlation with Joint Committee on Powder Diffraction Standards database (Cardno: 00-047-1049). In the XRD spectra, peaks at $37.1^{\circ}$ and $43.9^{\circ}$ correspond to miller indices (111) and (200) was found to be very high intense confirms the Face Centred Cubic (FCC) nature of crystal. The average particle size of NiO NPs was observed to $38 \mathrm{~nm}$ which was calculated based on Debye - Scherrer equation. The presence of oxygen spaces in the crystal and due to the local lattice disorder in the sample, the XRD spectra show broad peaks (Prabhu et al., 2013). The results of the XRD analysis proves that the NiO NPs formed by the reduction of $\mathrm{Ni}^{2+}$ by $S$. maritima, leaf aqueous extract was crystalline with an average particle size of $36 \mathrm{~nm}$ and this is in a good argument with the earlier reported reports (Hou et al., 2005; Pandian et al., 2015).

\subsection{Anti-bacterial activity study}

The NiO NPs synthesized in the study were found to be inhibiting the growth of the gram-positive and gram-negative pathogenic bacteria studied. The growth inhibition activity of the aqueous plant extract was also studied against the selected bacteria and found that the zone of inhibition was observed against all the bacteria. The zone of inhibition of the synthesized NPs was found to be more than the aqueous extract. At the lowest concentration of $10 \mu \mathrm{g} / \mathrm{mL}$ zone of inhibition of the aqueous plant extract was observed to be almost nil except for E.coli, where a very less zone of inhibition was observed. But the zone of inhibition at the said concentration for the synthesized NiO NPs was observed to be significant and a prominent zone was identified. At high concentration, significant zones were observed for both aqueous extract and the synthesized NiO NPs but the zone of inhibition was more enhanced for the NiO NPs than the aqueous extract. The control distilled water did not show any inhibition zone; hence it can be confirmed from the results of this study that the NiO NPs synthesized using $S$. maritima, leaf aqueous extract have very higher inhibition activity against the selected pathogenic bacteria in the study. The inhibition results of the current study have been given in table 1 and agar plates showing the zone of inhibition were sown in figure 7 .

\subsection{DPPH radical scavenging assay}

The antioxidant activity of the synthesized NiO NPs and the standard ascorbic acid was measured by DPPH free radical scavenging assay. The results were compared by using $50 \%$ radical inhibition concentration (IC 50) of each sample studied. The IC 50 values of the synthesized NiO NPs were observed

Table 1 Bacterial growth inhibition study results

\begin{tabular}{|ccccccccc|} 
& & \multicolumn{4}{c|}{ Zone of Inhibition observed in mm for the concentration studied } \\
\cline { 3 - 9 } S No & Bacteria Name & \multicolumn{2}{c}{$\begin{array}{c}10 \mu \mathrm{g} / \mathrm{mL} \\
\text { Aqueous leaf } \\
\text { extract }\end{array}$} & NiO NPs & $\begin{array}{c}\text { Aqueous leaf } \\
\text { extract }\end{array}$ & NiO NPs & $\begin{array}{c}\text { Aqueous leaf } \\
\text { extract }\end{array}$ & NiO NPs \\
\hline 1 & B. subtilis & No zone & 2.1 & 2.8 & 7.8 & 6.9 & 15.4 \\
\hline 2 & S.aureus & No zone & 2.4 & 3.1 & 8.4 & 6.3 & 13.7 \\
\hline 3 & E.coli & 2.8 & 3.7 & 5.9 & 9.5 & 11.7 & 18.1 \\
\hline 4 & P.aeruginosa & No zone & 2.8 & 4.6 & 9.1 & 9.8 & 17.6 \\
\hline
\end{tabular}

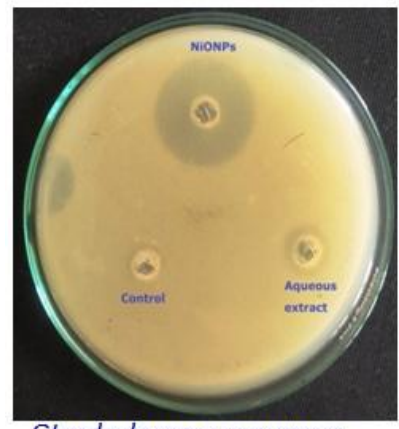

Staphylococcus aureus

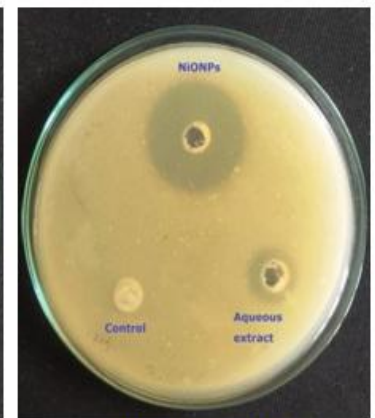

Bacillus subtilis

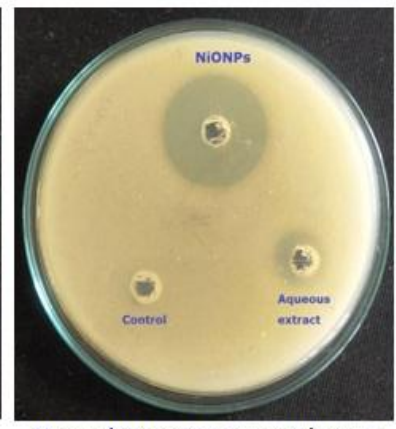

Pseudomonas aeruginosa

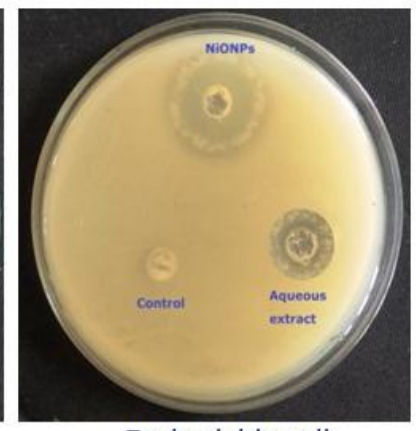

Escherichia coli

Figure 7 Anti-bacterial activity of aqueous leaf extract, synthesized NiO NPs and distilled water at a concentration of $50 \mu \mathrm{g} / \mathrm{mL}$ by agar plate well diffusion method

Journal of Experimental Biology and Agricultural Sciences http://www.jebas.org 
Table 2 DPPH radical scavenging assay results

\begin{tabular}{|c|c|c|c|c|}
\hline \multirow{2}{*}{ S No } & \multirow{2}{*}{ Concentration $(\mu \mathrm{g} / \mathrm{mL})$} & \multicolumn{3}{|c|}{ \% DPPH Inhibition } \\
\hline & & Ascorbic Acid & Aqueous extract & $\mathrm{NiO}$ NPs \\
\hline 1 & 5 & 7.16 & 0.63 & 3.46 \\
\hline 2 & 10 & 10.78 & 1.78 & 5.66 \\
\hline 3 & 15 & 24.25 & 4.11 & 12.15 \\
\hline 4 & 20 & 38.33 & 9.46 & 24.07 \\
\hline 5 & 25 & 60.70 & 18.95 & 37.57 \\
\hline 6 & 30 & 77.89 & 26.67 & 54.01 \\
\hline 7 & 35 & 91.69 & 35.14 & 72.20 \\
\hline 8 & 40 & 96.35 & 44.14 & 82.99 \\
\hline
\end{tabular}

$28.01 \mu \mathrm{g} / \mathrm{mL}$ and it was reported very close to the standard ascorbic acid $(22.19 \mu \mathrm{g} / \mathrm{mL})$ whereas the IC 50 of the aqueous plant leaf extract was found to be $47.30 \mu \mathrm{g} / \mathrm{mL}$. The results of the DPPH radical inhibition study confirm that the synthesized $\mathrm{NiO}$ NPs enhanced the free radical inhibition activity (Table 2).

A simple one-pot green synthesis of stable NiO NPs using $S$. maritima aqueous plant leaf extract at room temperature was reported in this study. The time of the bio-reduction and stability of the obtained NPs with no external stabilizers/reducing agents proves that the synthesis method is efficient.

\section{Conclusion}

The synthesized NPs were characterized for their anti-oxidant and anti-microbial activity and reported potential anti-oxidant and antibacterial agents. It proves an eco-friendly, rapid synthesis, costeffective and efficient mechanism of the synthesis of NiO NPs. Therefore, this reaction pathway satisfies all the conditions of a standard green chemical process.

\section{Abbreviations}

NiONPs: Nickel oxide nanoparticles; NiO: Nickel oxide; NPs: Nanoparticles; UV-Vis: UV-Visible spectrophotometry; XRD: Xray diffraction; FT- IR: Fourier transform infrared spectroscopy; SEM: Scanning electron microscopy.

\section{References}

Akintola AO, Kehinde BD, Ayoola PB, Adewoyin AG, Adedosu OT, Ajayi JF, Ogunsona SB (2020) Antioxidant properties of silver nanoparticles biosynthesized from methanolic leaf extract of Blighia sapida. IOP Conference Series: Materials Science and Engineering 805: 012004. doi:10.1088/1757-899X/805/1/012004.

Anandgaonker P, Kulkarni G, Gaikwad S, Rajbhoj A (2019) Synthesis of $\mathrm{TiO} 2$ nanoparticles by electrochemical method and their antibacterial application. Arabian Journal of Chemistry 2(8): 1815-1822.

Bandaranayake WM (2002) Bioactivities, bioactive compounds and chemical constituents of mangrove plants. Wetlands Ecology and Management 10:421-452.

Cristino AF, Matias IAS, Bastos DEN, Galhano SR, Ribeiro APC, Martins LMDRS (2020) Glycerol Role in Nano Oxides Synthesis and Catalysis. Catalysts 10(12): 1406.

Ebin B (2018) Simple Preparation of Ni and NiO Nanoparticles Using Raffinate Solution Originated from Spent NiMH Battery Recycling. Journal of Inorganic and Organometallic Polymers and Materials 28: 2554-2563

Evanoff DD, Chumanov G (2005) Synthesis and optical properties of silver nanoparticles andarrays. ChemPhysChem 6: 1221-1223.

Gokce EH, Korkmaz E, Tuncay Tanrıverdi S, Dellera E, Sandri G, Bonferoni MC, Ozer O (2012) a comparative evaluation of coenzyme Q10-loaded liposomes and solid lipid nanoparticles as dermal antioxidant carriers. International Journal of Nanomedicine 7: 5109-5117.

Haider A, Ijaz M, Ali S, Imran M, Majeed H, Shahzadi I, Ali MM, Khan JA (2020) Green Synthesized Phytochemically (Zingiber officinale and Allium sativum) Reduced Nickel Oxide Nanoparticles Confirmed Bactericidal and Catalytic Potential. Nanoscale Research Letters 15(1):50.

Hou Y, Kondoh H, Ohta T, Gao S (2005) Size-controlled synthesis of nickel nanoparticles. Applied Surface Science 241: 218-221.

Huston M, DeBella M, DiBella M, Gupta A (2021) Green Synthesis of Nanomaterials. Nanomaterials 11(8): 2130. 
Ibrahim K, Khalid S, Idrees K (2019) Nanoparticles: Properties, applications and toxicities. Arabian Journal of Chemistry 12(7): 908-931.

Khalid N, Munetaka O, Raquel D, Mohammed A, Kityk IV, Mosto B (2011) Nanoscale synthesis and optical features of metallic nickel nanoparticles by wet chemical approaches. Journal of Alloys and Compounds 509: 5882-5886.

Mohammed AA, Olajire AA (2020) Green synthesis of nickel oxide nanoparticles and studies of their photocatalytic activity in degradation of polyethylene films. Advanced Powder Technology 31(1): 211-218.

Mude N, Ingle A, Gade A, Rai M (2009) Synthesis of silver nanoparticles using callus extract of Carica papaya-a first report. Journal of Plant Biochemistry and Biotechnology 18:83-86.

Nayak B, Roy S, Roy M, Mitra A, Karak AK (2018) Phytochemical, Antioxidant and Antimicrobial Screening of Suaeda maritima L (Dumort) against Human Pathogens and Multiple Drug Resistant Bacteria. Indian Journal of Pharmaceutical Sciences 80(1):26-35.

Palasantzas G, Koch SA, Vsytavel T, Th J, De Hosson M (2008) Opportunities from the nanoworld: gas phase nanoparticles. Journal of Alloys and Compounds 449: 237-241.

Pandian CJ, Palanivel R, Dhananasekaran S (2015) Green synthesis of nickel nanoparticles using Ocimum sanctum and their application in dye and pollutant adsorption. Chinese Journal of Chemical Engineering 23(8): 1307-1315.

Pasupuleti VR, Prasad T, Shiekh RA (2013) Biogenic silver nanoparticles using Rhinacanthus nasutus leaf extract, synthesis, spectral analysis, and antimicrobial studies. International Journal of Nanomedicine 8: 3355-3364.

Patra JK, Dhal NK, Thatoi HN (2011) In vitro bioactivity and phytochemical screening of Suaeda maritima (Dumort): A mangrove associate from Bhitarkanika, India. Asian Pacific Journal of Tropical Medicine 4(9): 727-734.

Prabhu YT, Rao KV, Kumar VSS, Kumari BS (2013) Synthesis of $\mathrm{ZnO}$ nanoparticles by a novel surfactant assisted amine combustion method. Advances in Nanoparticles 2(1):45.

Ravi KS, Gnanadesigan M, Jacob IS, Kalaiarasi A (2011) Hepatoprotective and antioxidant properties of Suaeda maritima (L.) dumort ethanolic extract on concanavalin-A induced hepatotoxicity in rats. Indian Journal of Experimental Biology 49(6):455-60.

Singh J, Dutta T, Ki-Hyun K, Rawat M, Samddar P, Kumar P (2018) 'Green' synthesis of metals and their oxide nanoparticles: applications for environmental remediation, 1. Journal of Nanobiotechnology 16:84

Stark WJ, Stoessel PR, Wohlleben W, Hafner A (2015) Industrial applications of nanoparticles. Chemical Society Reviews 44: 5793-5805.

Suresh S, Saravanan P, Jayamoorthy K, Kumar SA, Karthikeyan S (2016) Development of silane grafted $\mathrm{ZnO}$ core shell nanoparticles loaded diglycidyl epoxy nanocomposites film for antimicrobial applications. Materials Science and Engineering C 64: 286-292.

Taiba N, Tayyiba D (2021) The role of some important metal oxide nanoparticles for wastewater and antibacterial applications: A review. Environmental Chemistry and Ecotoxicology 3: 59-75.

Wang T, Jin X, Chen Z, Megharaj M, Naidu R (2014) Green synthesis of $\mathrm{Fe}$ nanoparticles using eucalyptus leaf extracts for treatment of eutrophic waste water. Science of the Total Environment $466: 210-213$.

Zahra S, Alireza A, Hasan AH, Mehrdad K, Majid D (2021) Green-based bio-synthesis of nickel oxide nanoparticles in Arabic gum and examination of their cytotoxicity, photocatalytic and antibacterial effect. Green Chemistry Letters and Reviews 14(2): 404-414. 